\title{
EFFECT OF WEEDING REGIME ON THE WEED INFESTATION AND CROP PERFORMANCE OF TRANSPLANT AMAN RICE
}

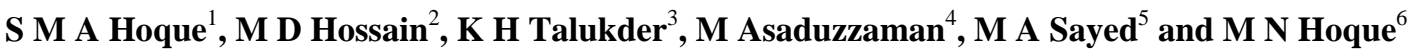

\begin{abstract}
An experiment was carried out at the Agronomy Field Laboratory, Bangladesh Agricultural University, Mymenshingh to study the effect of weeding regime on the weed infestation and crop performance on transplant aman rice. The treatments included 10 weeding regime - i) no weeding, ii) one hand weeding at 21 DAT, iii) two hand weeding at 21 and 42 DAT, iv) three hand weeding at 21, 42 and 63 DAT, v) Ronstar 25 EC @ $2.01 \mathrm{ha}^{-1}$ at pre-emergence, vi) 2, 4-D amine @ $1.841 \mathrm{ha}^{-1}$ at 42 DAT, vii) Ronstar 25 EC @ $2.01 \mathrm{ha}^{-1}$ at Pre-emergence + 2, 4-D amine @ $1.841 \mathrm{ha}^{-1}$ at 42 DAT, viii) Ronstar 25 EC @ $2.01 \mathrm{ha}^{-1}$ at Pre-emergence + one hand weeding at 42 DAT, ix) one hand weeding at $21 \mathrm{DAT}+2.4-\mathrm{D}$ amine @ $1.841 \mathrm{ha}^{-1}$ at $42 \mathrm{DAT}$ and X) weed free. The experiment was laid out in a randomized complete block design with four replications. Weeding regime had significant effect on weed density and dry weight. Weed density and dry weight were significantly highest in the crop not weeded at all and lowest in Ronstar 25 EC @ 2.01 ha $^{-1}+$ one hand weeding. Plant height, total number of tillers hill ${ }^{-1}$, number of ear bearing tillers hill $^{-1}$, number of non ear bearing tillers hill ${ }^{-1}$, number of grains panicle ${ }^{-1}$,number of sterile spike lets panicle ${ }^{-1}$, grain yield, straw yield, biological yield and harvest index were significantly affected by weeding regime. Ronstar 25 EC @ 2.01 ha $^{-1}+$ one hand weeding controlled the weeds to the greatest extent and produced the yield and yield attributes similar to those in weed free treatment.
\end{abstract}

Keywords: Weeding Regime, Weed Infestation, Crop Performance and Transplant Aman Rice.

\section{INTRODUCTION}

Transplant aman rice is the important rice crop of Bangladesh which covers about $33.40 \%$ of total rice area and contributes to $47.73 \%$ of the total rice production (BBS, 2008). But in this crop, yield is much lower than that of transplanted rice in other rice growing countries of the world. Severe weed infestation constitutes one of the reasons for such low yield (Mamun, 1988). The yield loss due to weed completion in transplant aman rice is $40 \%$ in Bangladesh (BRRI, 1981). In Bangladesh weeds are being controlled manually by hand pulling or by using simple tools like niranee, Japanese rice weeder etc. Usually, two to three hand weddings are done for growing a transplant rice crop depending upon the nature of weeds and their intensity of infestation. But, this method is very laborious, time consuming and expensive. The use of herbicide can help controlling weeds more easily and cheaply. Weed competition at early growth stage can be eliminated through pre-emergence herbicides and weeds growing at later crop growth stage can be controlled by post-emergence herbicides. Preemergence herbicides, post emergence herbicides or both if used in combination with hand weeding

\footnotetext{
1 Territory Sales Manager, Shetu Marketing Company(SEMCO), Dhaka, Bangladesh

2 Lecturer, Department of Agribusiness, Atish Dipankar University of Science and Technology(ADUST); Bangladesh

${ }^{3}$ Professor \& Dean, Faculty of Agriculture, Biological Science, Biotechnology \& Textile, Atish Dipankar University of Science and Technology(ADUST)

4 Executive Director, Bangladesh Science Foundation, Dhaka, Bangladesh

5 Scientific Officer, Genetics \& Plant Breeding Division, BRRI, Gazipur

6 Scientific Officer, Genetics \& Plant Breeding Division, BARI, Gazipur
} 
more efficient weed control may be achieved. It has been seen that combination of two methods are always better than single method that is only hand weeding or only chemical weeding. Moreover, repeated use of any single method makes an unimportant weed to establish as an important one. Efforts to suppress the seed infestation with simultaneous increase in crop production through improved cultivation require the introduction of use of herbicide. The replacement of manual weeding by herbicides or herbicides in combination with hand weeding would help to obtain higher crop productivity with less efforts and cost. The present study was, therefore, undertaken to study the effect of weeding regime on the weed infestation and crop performance of transplant aman rice.

\section{MATERIALS AND METHODS}

An Experiment was conducted at the Agronomy Field Laboratory, Bangladesh Agricultural University (BAU), Mymensingh to study the effect of weeding regime on the weed infestation and crop performance of transplant aman rice. The experiment was laid out in a randomized complete block design with four replications. The treatments included 10 weeding regime-i) no weeding, ii) One hand weeding at 21 DAT, iii) two hand weeding at 21 and 42 DAT, iv) three hand weeding at 21, 42 and 63 DAT, v) Ronstar 25 EC @ 2.01 ha $^{-1}$ at pre-emergence, vi) 2, 4-D amine @ 1.841 ha $^{-1}$ at 42 DAT, vii) Ronstar 25 EC@ 2.01 ha- $^{1}$ at pre- emergemce + 2, 4-D amine @ 1.841 ha $^{-1}$ at 42 DAT viii) Ronstar 25 EC@ 2.01 ha- $^{1}$ at pre- emergemce + one hand weeding at 42 DAT ix) One hand weeding at 21 DAT + 2, 4-D amine @ $1.841 \mathrm{ha}^{-1}$ at 42 DAT and $\mathrm{x}$ ) weed free. The area of unit plot was 0.0015 ha $(5 \mathrm{mx} 3 \mathrm{~m})$. The treatments were allocated in each block at random. The land was fertilized with 69, 18.86, 20, 10.8 and $3.6 \mathrm{~kg}$ of N, P, K,S and Zn ha ${ }^{-1}$ through urea, triple super phosphate, muriate of Potash, gypsum and zinc sulphate, respectively. The whole amount of triple super phosphate, muriate of potash, gypsum and zinc sulphate were applied as basal dose at final land preparation and the whole amount of urea was top dressed in three equal installments at 10, 30 and 55 DAT. The seedlings of BR11 ( Mukta) were transplanted on the well puddled unit plots maintaining a spacing of $25 \mathrm{~cm} \times 15$ $\mathrm{cm}$ (row to row distance $25 \mathrm{~cm}$ and hill to hill distance $15 \mathrm{~cm}$ ). Two seedlings were used per hill. Weeding was done as per the weeding regime. Irrigation was done when required. Proper preventive measures were taken to protect the crop from insect pests. The crop was harvested plot wise on 2 December 1999. Data were collected on the density and dry weight of the infesting weed species. Phytotoxicity of Ronstar 25 EC @ 2.01 ha $^{-1}$ and 2, 4-D amine @ 1.841 ha $^{-1}$ to experimental crop were determined by visual observations. Data on different crop characters were recorded at harvest of the crop. The mean differences of the analyzed data were adjudged as per Duncan's Multiple Range Test.

\section{RESULTS AND DISCUSSION}

The results of the present study have been presented in Tables 1-5. Twenty eight different weed species belonging to 13 families, of which 23 were annuals against 5 perennials, were found to infest the experimental crop (as observed in the crop receiving no weeding at flowering stage of rice plants). Of these species, 7 belonged to Gramineae, 6 to Cyperaceae, and 2 to each of the Amaranthaceae, Compositae, Commelinaceae and Euphorbiaccae. Only one species represented the rest of the 7 families- Pontederiaccae, Onagraceae, Oxalidaceae, Marsileaceae, Polygonaccae, Lythraceae and Convolvulaceae. Local name, scientific name, family, classification and important values (\%) of these weeds have been presented in Table1. The most important weed species in the experimental plots at flowering stage of rice plants was Angta ( Panicum repens). Other 4 most important weeds were Kanainala ( Murdania nudiflora), Matichaise ( Fimbristylis miliacea), Anguleeghash (Digitaria 
sanguinalis) and Nakphuli(Cyperus michelianus). These five weed species contributed $67.95 \%$ of the total weed dry weight. The remaining $32.05 \%$ of the total dry weight was contributed by the rest of the 23 weed species (Table 1). Keshnuti( Eclipta alba) was the least important weed species in the experimental field.

Weed density was significantly affected by methods of land preparation. The highest weed density was recorded in the plots prepared by country plough and the lowest in the plots prepared by tractor (Table 2). The weed density in the plots prepared by power tiller was in between that of the plots prepared by country plough and tractor. Weed density in the plots prepared by power tiller and tractor being statistically identical was significantly lower, $44.46 \%$ and $56.40 \%$ respectively, than that in the plots prepared by country plough. It is thought that the variation in the depth of ploughing might be responsible for such variation in the weed density. As already discussed, it may be seen from Table I that, compared to that in the plots ploughed by country plough, the depth of ploughing increaser by $32.98 \%$ in the plots prepared by power tiller and $76.77 \%$ in the plot prepared by tractor. In other wards, it may be said that, weed density decreased as the depth of ploughing increased. Similar results of reduced weed density with increased depth of ploughing due to tractor ploughing compared to power tiller ploughing was also reported by Kim et al (1975).

Table 1. Local name, scientific name, family, classification of weed types, and importance value (\%) of the weed species found growing in the plots receiving no weeding.

\begin{tabular}{|c|c|c|c|c|}
\hline Local name & Scientific name & Family & Classification & $\begin{array}{l}\text { Importance } \\
\text { Value (\%) }\end{array}$ \\
\hline 1. Angta & Panicum repens & Gramineae & Perennial, Grass & 42.51 \\
\hline 2. Kanainala & Murdania nudiflora & Commelinaceae & Annual; Broadleaf & 8.72 \\
\hline 3.Matichaise & Fimbristylis miliacea & Cyperaceae & Annual, Sedge & 7.09 \\
\hline 4.Anguleeghash & Digitaria Sanguinalis & Gramineae & Annual,Grass & 5.56 \\
\hline 5.Nakphuli & Cyperus michelianus & Cyperaceae & Annual,Sedge & 4.07 \\
\hline 6. Joina & Fimbristylis diphylla & Cyperaceae & Annual,Grass & 3.69 \\
\hline 7. Arail & Leersia hexandra & Gramineae & Annual,Grass & 2.78 \\
\hline 8. Chanchi & Alternathera sessilis & Amaranthaceae & Annual, Brodleaf & 2.53 \\
\hline 9. Panikachu & Monochoria hastata & Pontederiaceae & Annual, Brodleaf & 2.18 \\
\hline 10. Shusnishak & Marsilea crenata & Marsileaceae & Annual, Brodleaf & 2.02 \\
\hline 11. Chechra & Scirpus mucronatus & Cyperaceae & Perennial; Sedge & 1.94 \\
\hline 12. Panilong & Ludwigina prostrata & Onagraceae & Annual, Broadleaf & 1.70 \\
\hline 13. Khudeyshama & Echinochloa colonum & Gramineae & Annual; Grass & 1.42 \\
\hline 14. Durba & Cynodon dactylon & Gramineae & Annual; Grass & 1.32 \\
\hline 15. Kalmilata & Ipomea aquatica & Convolvulaceae & Perennial; Broadleaf & 1.28 \\
\hline 16. Panichaise & Eleocharis atropurpurea & Cyperaceae & Annual; Sedge & 1.22 \\
\hline 17. Amrulshak & Oxalis europaea & Oxalidaceae & Annual; Broadleaf & 1.11 \\
\hline 18. Chotodhudia & Euphorbia prostrata & Euphorbiaceae & Annual;Broadleaf & 1.04 \\
\hline 19. Acidghash & Ammania baccifera & Lythraceae & Aannual;Brodleaf & 0.99 \\
\hline 20. Panimarich & Polygonum orientale & Polygonaceae & Annual, Brodleaf & 0.95 \\
\hline 21. Kanaibashi & Commelina diffusa & Commelinaceae & Annual; Brodleaf & 0.94 \\
\hline 22. Malancha & Alternathera Philoxeroides & Amaranthaceae & Perennial, Brodleaf & 0.87 \\
\hline 23. Barashama & Echinochloa crus-galli & Gramineae & Annual; Grass & 0.86 \\
\hline 24. Helencha & Enhydra fluctuans & Compositae & Annual; Broadleaf & 0.78 \\
\hline 25. Nolchaise & Scirpus articulatus & Cyperaceae & Annual, Sedge & 0.75 \\
\hline 26. Chelaghash & Parapholis incurva & Gramineae & Annual; Grass & 0.71 \\
\hline 27. Baradhudia & Eupgorbia hirta & Euphorbiaceae & Annual; Broadleaf & 0.51 \\
\hline 28. Keshuti & Eclipta alba & Compositae & Annual; Broadleaf & 0.48 \\
\hline
\end{tabular}


Table 2. Effect of weeding regime on the weed density and weed dry weight.

\begin{tabular}{|c|c|c|}
\hline Weeding regime & $\begin{array}{l}\text { Weed density } \\
\left(\text { no.m }{ }^{-2}\right)\end{array}$ & $\begin{array}{l}\text { Weed dry weight } \\
\qquad\left(\mathrm{g} \mathrm{m}^{-2}\right)\end{array}$ \\
\hline No weeding $\left(\mathrm{W}_{0}\right)$ & $94.45 \mathrm{a}$ & $83.02 \mathrm{a}$ \\
\hline One hand weeding at $21 \mathrm{DAT}\left(\mathrm{W}_{1}\right)$ & 48.14b (-49.03)* & 53.03b (-36.12) \\
\hline Two hand weeding at 21 and 42 DAT $\left(\mathrm{W}_{2}\right)$ & 35.98b (-61.91) & 39.08bc(-52.93) \\
\hline Three hand weeding at 21, 42 and 63 DAT $\left(\mathrm{W}_{3}\right)$ & $30.96 b(-67.22)$ & $26.13 c d(-68.53)$ \\
\hline Ronstar 25 EC @ 2.01 ha $^{-1}$ at pre-emergence $\left(\mathrm{W}_{4}\right)$ & 43.66b (-53.77) & 18.43cde(-77.80) \\
\hline 2,4-D amine @ 1.841 ha $^{-1}$ at 42 DAT $\left(\mathrm{W}_{5}\right)$ & $51.46 \mathrm{~b}(-45.52)$ & $22.34 c d(-73.09)$ \\
\hline $\begin{array}{l}\text { Ronstar } 25 \text { EC @ } 2.01 \mathrm{ha}^{-1} \text { at pre-emergence + } \\
\text { 2,4-D amine @ } 1.841 \mathrm{ha}^{-1} \text { at } 42 \mathrm{DAT}\left(\mathrm{W}_{6}\right)\end{array}$ & 37.32b (-60.49) & 16.86de (-79.69) \\
\hline $\begin{array}{l}\text { Ronstar } 25 \text { EC @ } 2.01 \text { ha }^{-1} \text { at pre-emergence }+ \text { One hand } \\
\text { weeding at } 21 \text { DAT }\left(\mathrm{W}_{7}\right)\end{array}$ & 28.60b (-69.72) & 14.55de (-82.47) \\
\hline $\begin{array}{l}\text { One hand weeding at } 21 \mathrm{DAT}+2,4-\mathrm{D} \text { amine @ } \\
1.841 \mathrm{ha}^{-1} \text { at } 42 \mathrm{DAT}\left(\mathrm{W}_{8}\right)\end{array}$ & $32.27 \mathrm{~b}(-65.83)$ & 20.89cde(-74.83) \\
\hline Weed free $\left(\mathrm{W}_{9}\right)$ & $0.00 \mathrm{c}$ & $0.00 \mathrm{e}$ \\
\hline Sx & 5.688 & 5.261 \\
\hline $\mathrm{CV}(\%)$ & 27.17 & 34.29 \\
\hline Level of significance & 0.01 & 0.01 \\
\hline
\end{tabular}

In a column, figure having a common letter (s) do not differ significantly.

* Figures in the parentheses indicate percent decrease (-) compared to the value under no weeding in the respective column.

Weed density was significantly affected by weeding regime. The highest weed density was recorded in the crop not weeded at all and the lowest in Ronstar 25 EC @ $2.01 \mathrm{ha}^{-1}+$ one hand weeded crop (Table 2). Weed density in one hand weeding, two hand weeding, three hand weeding, Ronstar 25 EC @ $2.01 \mathrm{ha}^{-1}$, 2, 4-D amine @ $1.841 \mathrm{ha}^{-1}$, Ronstar $25 \mathrm{EC} @ 2.01 \mathrm{ha}^{-1}+$ one hand weeding and one hand weeding + 2,4-D amine @ 1.841 ha $^{-1}$ being statistically identical was significantly lower than that in no weeding. This, in other words means, that, the weed control measures, singly or in combination, as included in this study, were of similar efficiency. The effect was similar in reducing the weed population whether the crop was hand weeded once, twice or thrice, or chemically weeded by Ronstar 25 EC @ 2.01 ha $^{-1}$ or 2, 4-D amine @ 1.841 ha $^{-1}$ or both in combination or by any one of these two herbicides in combination with one hand weeding. Nolchaise, Barashama, Durba, Kanatbashi, Chotodlhudia and Baradhnudia was totally controlled by hand weeding whatever might be the frequency (Table 3). In case of all other weeds, the extent of reduction of density increased with the increase in the frequency of hand weeding, the highest being in the thrice hand weeding. Ronstar 25 EC @ 2.01 ha $^{-1}$ totally failed to control Nakphuli, Nolchaise, Arail and Chelaghash. The density of Angta, Kanainala, Matichaise and Anguleeghash which were among the four most important weeds were reduced to the extent of 56-72\% only by Ronstar 25 EC @ 2.01 ha $^{-1}$. Nakphuli, Panichaise, Nolchaise, Khudeyshama and Arial could not be controlled by 2, 4-D amine @ 1.841 ha $^{-1}$. Angta, the most important weed in the experimental field, was controlled only by $51.28 \%$ by 2,4-D amine @ $1.841 \mathrm{ha}^{-1}$.The extent of control of Matichaise by 2,4-D amine @ $1.841 \mathrm{ha}^{-1}$ was also very poor. However, it reduced the density of Anguleeghash and Kanainala by about 83 and 86\%, respectively. Ronstar 25 EC @ 2.01 ha $^{-1}+$ 2,4-D amine @ 1.841 ha $^{-1}$ through failed to control Nakphuli, Nolchaise, Arail and Chelaghash, it totally controlled Panichaise, Barashama, Durba, Malancha, Keshuti, Helencha, Kanaibashi, Chhotodhudia, Baradhudia, Panikachu, Panilong, Amrulshak, Shusnishak, Panimarich, Acidghash and Kalmilata. It reduced the density of Joina, Anguleeghash, Chanchi and Kanainala to the extent of more that 80\% (Table 3). Ronstar 25 EC @ 2.0 lha $^{-1}+$ one hand weeding controlled Angta, Anguleeghash. Matichaise and Kanainala by around 70-74\%. It controlled Nakphuli by only 41.79\%. It, however, totally controlled Panichaise, Nolchaise, 
Barashama, Durba, Malancha, Keshuti, Helencha, Kanaibashi, Chhotodhudia, Baradhudia, Panikachu, Panilong, Amrulshak, Shusnishak, Acidghash and Kalmilata. 
Table 3. Effect of weeding regime on the density of individual weed species

\begin{tabular}{|c|c|c|c|c|c|c|c|c|c|c|}
\hline \multirow{2}{*}{$\begin{array}{l}\text { Weeding } \\
\text { regime }\end{array}$} & \multicolumn{10}{|c|}{ Weed density ( no.m -2) } \\
\hline & Joina & Matichaise & Nakphuli & Panichaise & Nolchaise & Chechra & Angta & Khudeyshama & Anguleeghash & Barashama \\
\hline $\mathrm{W}_{0}$ & 3.92 & 9.91 & 3.47 & 1.43 & 1.01 & 2.45 & 37.48 & 1.11 & 6.61 & 1.19 \\
\hline $\mathrm{W}_{1}$ & $\begin{array}{l}2.11 \\
(-46.17)\end{array}$ & $\begin{array}{l}5.34 \\
(-46.12)\end{array}$ & $\begin{array}{l}1.80 \\
(-48.13)\end{array}$ & $\begin{array}{l}0.77 \\
(-46.15)\end{array}$ & - & $\begin{array}{l}1.32 \\
(-46.12)\end{array}$ & $\begin{array}{l}20.42 \\
(-45.52)\end{array}$ & $\begin{array}{l}0.59 \\
(-46.85)\end{array}$ & $\begin{array}{l}3.71 \\
(-43.87)\end{array}$ & - \\
\hline $\mathrm{W}_{2}$ & $\begin{array}{l}1.62 \\
(-58.67)\end{array}$ & $\begin{array}{l}3.95 \\
(-60.14)\end{array}$ & $\begin{array}{l}1.40 \\
(-59.65)\end{array}$ & $\begin{array}{l}0.58 \\
(-59.44)\end{array}$ & - & $\begin{array}{l}0.99 \\
(-59.59)\end{array}$ & $\begin{array}{l}15.44 \\
(-58.80)\end{array}$ & $\begin{array}{l}0.45 \\
(-59.46)\end{array}$ & $\begin{array}{l}2.67 \\
(-59.61)\end{array}$ & - \\
\hline $\mathrm{W}_{3}$ & $\begin{array}{l}1.42 \\
(-63.78)\end{array}$ & $\begin{array}{l}3.57 \\
(-63.98)\end{array}$ & $\begin{array}{l}1.25 \\
(-63.98)\end{array}$ & $\begin{array}{l}0.52 \\
(-63.64)\end{array}$ & - & $\begin{array}{l}0.88 \\
(-64.08)\end{array}$ & $\begin{array}{l}13.65 \\
(-63.58)\end{array}$ & $\begin{array}{l}0.41 \\
(-63.06)\end{array}$ & $\begin{array}{l}2.38 \\
(-63.99)\end{array}$ & - \\
\hline $\mathrm{W}_{4}$ & $\begin{array}{l}1.56 \\
(-60.20)\end{array}$ & $\begin{array}{l}3.66 \\
(-63.07)\end{array}$ & $\begin{array}{l}4.14 \\
(+19.31)\end{array}$ & - & $\begin{array}{l}1.42 \\
(+40.59)\end{array}$ & $\begin{array}{l}2.05 \\
(-16.33)\end{array}$ & $\begin{array}{l}16.49 \\
(-56.00)\end{array}$ & $\begin{array}{l}0.95 \\
(-14.41)\end{array}$ & $\begin{array}{l}1.89 \\
(-71.41)\end{array}$ & - \\
\hline $\mathrm{W}_{5}$ & $\begin{array}{l}3.71 \\
(-5.36)\end{array}$ & $\begin{array}{l}7.32 \\
(-26.14)\end{array}$ & $\begin{array}{l}5.06 \\
(+45.82)\end{array}$ & $\begin{array}{l}2.47 \\
(+72.73)\end{array}$ & $\begin{array}{l}2.37 \\
(+134.65)\end{array}$ & $\begin{array}{l}2.39 \\
(-2.45)\end{array}$ & $\begin{array}{l}18.26 \\
(-51.28)\end{array}$ & $\begin{array}{l}1.98 \\
(+78.38)\end{array}$ & $\begin{array}{l}1.17 \\
(-82.30)\end{array}$ & - \\
\hline $\mathrm{W}_{6}$ & $\begin{array}{l}0.46 \\
(-88.27)\end{array}$ & $\begin{array}{l}3.47 \\
(-64.98)\end{array}$ & $\begin{array}{l}4.16 \\
(+19.88)\end{array}$ & - & $\begin{array}{l}1.88 \\
(+86.14)\end{array}$ & $\begin{array}{l}1.86 \\
(-24.08)\end{array}$ & $\begin{array}{l}16.52 \\
(-55.92)\end{array}$ & $\begin{array}{l}1.07 \\
(-3.60)\end{array}$ & $\begin{array}{l}1.14 \\
(-82.75)\end{array}$ & - \\
\hline $\mathrm{W}_{7}$ & $\begin{array}{l}0.48 \\
(-87.76)\end{array}$ & $\begin{array}{l}2.60 \\
(-73.76)\end{array}$ & $\begin{array}{l}2.02 \\
(-41.79)\end{array}$ & - & - & $\begin{array}{l}1.69 \\
(-31.02)\end{array}$ & $\begin{array}{l}11.28 \\
(-69.90)\end{array}$ & $\begin{array}{l}0.96 \\
(-13.51)\end{array}$ & $\begin{array}{l}1.85 \\
(-72.01)\end{array}$ & - \\
\hline $\mathrm{W}_{8}$ & $\begin{array}{l}1.67 \\
(-57.40)\end{array}$ & $\begin{array}{l}2.97 \\
(-70.03)\end{array}$ & $\begin{array}{l}1.80 \\
(-48.13)\end{array}$ & $\begin{array}{l}1.61 \\
(+12.59)\end{array}$ & - & $\begin{array}{l}1.27 \\
(-48.16)\end{array}$ & $\begin{array}{l}14.70 \\
(-60.78)\end{array}$ & $\begin{array}{l}0.79 \\
(-28.83)\end{array}$ & $\begin{array}{l}1.11 \\
(-83.21)\end{array}$ & - \\
\hline$\overline{W_{9}}$ & - & - & - & - & - & - & - & - & - & - \\
\hline
\end{tabular}

Table 3. Continued

\begin{tabular}{|c|c|c|c|c|c|c|c|c|c|c|}
\hline \multirow{2}{*}{$\begin{array}{l}\text { Weeding } \\
\text { regime }\end{array}$} & \multicolumn{10}{|c|}{ Weed density ( no.m -2) } \\
\hline & Arial & Chelaghash & Durba & Chanchi & Malancha & Keshuti & Helencha & Kanainala & Kanaibashi & Chhotodhudia \\
\hline $\mathrm{W}_{0}$ & 2.79 & 1.40 & 1.29 & 2.36 & 1.34 & 0.61 & 0.98 & 1.96 & 0.68 & 1.36 \\
\hline $\mathrm{W}_{1}$ & $\begin{array}{l}1.53 \\
(-45.16)\end{array}$ & $\begin{array}{l}0.79 \\
(-43.57)\end{array}$ & - & $\begin{array}{l}1.57 \\
(-33.47)\end{array}$ & $\begin{array}{l}0.60 \\
(-55.22)\end{array}$ & $\begin{array}{l}0.29 \\
(-52.46)\end{array}$ & $\begin{array}{l}0.57 \\
(-41.84)\end{array}$ & $\begin{array}{l}1.31 \\
(-33.16)\end{array}$ & - & - \\
\hline $\mathrm{W}_{2}$ & $\begin{array}{l}1.13 \\
(-59.50)\end{array}$ & $\begin{array}{l}0.56 \\
(-60.00)\end{array}$ & - & $\begin{array}{l}1.18 \\
(-50.00)\end{array}$ & $\begin{array}{l}0.45 \\
(-66.42)\end{array}$ & $\begin{array}{l}0.23 \\
(-62.30)\end{array}$ & $\begin{array}{l}0.42 \\
(-57.14)\end{array}$ & $\begin{array}{l}0.98 \\
(-50.00)\end{array}$ & - & - \\
\hline $\mathrm{W}_{3}$ & $\begin{array}{l}0.99 \\
(-64.52)\end{array}$ & $\begin{array}{l}0.50 \\
(-64.29)\end{array}$ & - & $\begin{array}{l}1.05 \\
(-55.51)\end{array}$ & - & - & $\begin{array}{l}0.38 \\
(-61.22)\end{array}$ & $\begin{array}{l}0.88 \\
(-55.10)\end{array}$ & - & - \\
\hline $\mathrm{W}_{4}$ & $\begin{array}{l}4.27 \\
(+53.05)\end{array}$ & $\begin{array}{l}3.19 \\
(+127.86)\end{array}$ & $\begin{array}{l}0.36 \\
(-72.09)\end{array}$ & $\begin{array}{l}1.41 \\
(-40.25)\end{array}$ & $\begin{array}{l}0.33 \\
(-67.00)\end{array}$ & - & - & $\begin{array}{l}0.55 \\
(-71.94)\end{array}$ & - & - \\
\hline $\mathrm{W}_{5}$ & $\begin{array}{l}3.13 \\
(+12.19)\end{array}$ & $\begin{array}{l}1.13 \\
(-19.29)\end{array}$ & $\begin{array}{l}0.13 \\
(-89.92)\end{array}$ & $\begin{array}{l}0.28 \\
(-88.14)\end{array}$ & - & $\begin{array}{l}0.18 \\
(-70.49)\end{array}$ & -- & $\begin{array}{l}0.28 \\
(-85.71)\end{array}$ & - & - \\
\hline $\mathrm{W}_{6}$ & $\begin{array}{l}4.10 \\
(+46.95)\end{array}$ & $\begin{array}{l}2.06 \\
(+47.14)\end{array}$ & - & $\begin{array}{l}0.32 \\
(-86.44)\end{array}$ & - & - & - & $\begin{array}{l}0.26 \\
(-86.73)\end{array}$ & - & - \\
\hline $\mathrm{W}_{7}$ & 2.17 & 2.12 & - & 2.06 & - & - & - & 0.50 & - & - \\
\hline
\end{tabular}




\begin{tabular}{|c|c|c|c|c|c|c|c|c|c|c|}
\hline \multirow{2}{*}{$\begin{array}{l}\text { Weeding } \\
\text { regime }\end{array}$} & \multicolumn{10}{|c|}{ Weed density ( no.m -2) } \\
\hline & Arial & Chelaghash & Durba & Chanchi & Malancha & Keshuti & Helencha & Kanainala & Kanaibashi & Chhotodhudia \\
\hline & $(-22.22)$ & $(+51.43)$ & & $(-12.71)$ & & & & $(-74.49)$ & & \\
\hline $\mathrm{W}_{8}$ & $\begin{array}{l}2.99 \\
(+7.17)\end{array}$ & $\begin{array}{l}1.06 \\
(-24.29)\end{array}$ & - & $\begin{array}{l}0.31 \\
(-86.86)\end{array}$ & - & $\begin{array}{l}0.12 \\
(-80.33)\end{array}$ & - & $\begin{array}{l}0.26 \\
(-86.73)\end{array}$ & - & - \\
\hline $\mathrm{W}_{9}$ & - & - & - & - & - & - & - & - & - & - \\
\hline
\end{tabular}

\section{Table 3. Continued}

\begin{tabular}{|c|c|c|c|c|c|c|c|c|}
\hline \multirow{2}{*}{$\begin{array}{l}\text { Weeding } \\
\text { regime }\end{array}$} & \multicolumn{8}{|c|}{ Weed density ( no.m -2) } \\
\hline & Baradhudia & Panikachu & Panilong & Amrulshak & Shusnishak & Panimarch & Acidghash & Kalmilata \\
\hline $\mathrm{W}_{0}$ & 0.57 & 1.42 & 1.61 & 1.76 & 3.36 & 1.63 & 0.51 & 0.28 \\
\hline $\mathrm{W}_{1}$ & - & $\begin{array}{l}0.72 \\
(-49.30)\end{array}$ & $\begin{array}{l}0.95 \\
(-40.99)\end{array}$ & $\begin{array}{l}0.94 \\
(-46.59)\end{array}$ & $\begin{array}{l}1.55 \\
(-53.87)\end{array}$ & $\begin{array}{l}0.93 \\
(-42.94)\end{array}$ & $\begin{array}{l}0.17 \\
(-66.67)\end{array}$ & $\begin{array}{l}0.15 \\
(-46.43)\end{array}$ \\
\hline $\mathrm{W}_{2}$ & - & $\begin{array}{l}0.56 \\
(-60.56)\end{array}$ & $\begin{array}{l}0.72 \\
(-55.28)\end{array}$ & $\begin{array}{l}0.75 \\
(-57.39)\end{array}$ & $\begin{array}{l}1.25 \\
(-62.80)\end{array}$ & $\begin{array}{l}0.65 \\
(-60.12)\end{array}$ & - & - \\
\hline $\mathrm{W}_{3}$ & - & $\begin{array}{l}0.51 \\
(-64.08) \\
\end{array}$ & $\begin{array}{l}0.60 \\
(-62.73) \\
\end{array}$ & $\begin{array}{l}0.63 \\
(-64.20) \\
\end{array}$ & $\begin{array}{l}1.16 \\
(-65.48) \\
\end{array}$ & $\begin{array}{l}0.17 \\
(-89.87) \\
\end{array}$ & - & - \\
\hline $\mathrm{W}_{4}$ & - & - & - & $\begin{array}{l}0.52 \\
(-70.45)\end{array}$ & $\begin{array}{l}0.33 \\
(-90.18)\end{array}$ & $\begin{array}{l}0.53 \\
(-67.48)\end{array}$ & - & - \\
\hline $\mathrm{W}_{5}$ & - & - & $\begin{array}{l}0.55 \\
(-65.84) \\
\end{array}$ & - & $\begin{array}{l}0.53 \\
(-84.23) \\
\end{array}$ & $\begin{array}{l}0.29 \\
(-82.21) \\
\end{array}$ & - & $\begin{array}{l}0.09 \\
(-67.86) \\
\end{array}$ \\
\hline $\mathrm{W}_{6}$ & - & - & - & - & - & - & - & - \\
\hline $\mathrm{W}_{7}$ & - & - & - & - & - & $\begin{array}{l}0.87 \\
(-46.63) \\
\end{array}$ & - & - \\
\hline $\mathrm{W}_{8}$ & - & - & $\begin{array}{l}0.52 \\
(-67.70)\end{array}$ & - & $\begin{array}{l}0.50 \\
(-85.12)\end{array}$ & $\begin{array}{l}0.56 \\
(-65.64)\end{array}$ & - & - \\
\hline $\mathrm{W}_{9}$ & - & - & - & - & - & - & - & - \\
\hline
\end{tabular}

Figures in the parentheses indicate percent decrease (-) or increase (+) in comparison to the value against no weeding treatment in the respective column. $\mathrm{W}_{0}=\mathrm{No}$ weeding , $\mathrm{W}_{1}=\mathrm{O}_{\mathrm{O} \text { - hand }}$ weeding at $21 \mathrm{DAT}, \mathrm{W}_{2}=$ Two hand weeding at 21 and 42 DAT, $\mathrm{W}_{3}=$ Three hand weeding at 21, 42 and 63 DAT, $\mathrm{W}_{4}=$ Ronstar 25 EC @ 2.01 ha $^{-1}$ at pre-emergence, $\mathrm{W}_{5}=2$, $4-\mathrm{D}$ amine @ 1.84 I ha ${ }^{-1}$ at $42 \mathrm{DAT}$ $\mathrm{W}_{6}=$ Ronstar 25 EC @ 2.01 ha $^{-1}$ at pre-emergence +2, 4-D amine @ $1.84 \mathrm{I} \mathrm{ha}^{-1}$ at 42 DAT, $\mathrm{W}_{7}=$ Ronstar 25 EC @ 2.0 lha ${ }^{-1}$ at pre-emergence + One and weeding at 42 DAT, W. $=$ One weeding at 21 DAT + 2,4-amine @ 1.841 ha $^{-1}$ at 42 DAT and W $\mathrm{W}_{9}=$ Weed Free. 
One hand weeding +2,4-D amine @ 1.841 ha $^{-1}$ controlled Angta by about 61\%, Kanainala by about $87 \%$, Matichaise by about $70 \%$, Anguleeghash by about $83 \%$ and Nakphuli by about $48 \%$. It, however, controlled Chanchi, Keshuti and Shusnishak by more than $80 \%$.Weed dry weight was significantly affected by weeding regime. It was significantly highest in no weeding and significantly lowest in Ronstar 25 EC @ 2.01 ha $^{-1}+$ one hand weeding. This means that Ronstar 25 EC @2.0 $1 \mathrm{ha}^{-1}+$ one hand weeding controlled the weeds to the greatest extent. However, the weed dry weight in this treatment was statistically identical with that in three hand weeding , Ronstar 25 EC @ 2.01 ha ${ }^{-1}$ 2,4-D amine @ 1.841 ha $^{-1}$, Ronstar 25 EC @ 2.01 ha $^{-1}+$ 2, 4-D amine @ $1.841 \mathrm{ha}^{-1}$ treatment or one hand weeding + 2,4-D amine @ $1.841 \mathrm{ha}^{-1}$ (Table 2). This, in other words means, that , the weed control efficiency of these weeding treatments was similar. However, weed dry weight in two hand weeding being statistically identical with that in one hand weeding was significantly lower than that in Ronstar 25 EC @ 2.01 ha $^{-1}+$ one hand weeding. This means that, weed control efficiency of two hand weeding was similar to that of one hand weeding but significantly lower than that of Ronstar 25 EC @ 2.01 ha $^{-1}+$ one hand weeding.

In response to hand weeding, the dry weight of individual weeds also showed a trend of reduction similar to that of density of individual weeds, that is reduction was highest in three hands weeding and lowest in one hand weeding (Table 4). Ronstar 25 EC @2.01 ha ${ }^{-1}+$ one hand weeding reduced dry weight of Angta, Matichaise, Nakphuli and Kanainala to the greatest extent. The dry weight of Anguleeghasi was reduced by about 84 to 91\% by Ronstar 25 EC @ 2.01 ha $^{-1}$, 2, 4-D amine @ $1.841 \mathrm{ha}^{-1}$, Ronstar 25 EC @ $2.01 \mathrm{ha}^{-1}+$ one hand weeding and one hand weeding + 2,4-D amine @ $1.841 \mathrm{ha}^{-1}$. Ronstar 25 EC @ $2.01 \mathrm{ha}^{-1}$ 2, 4-D amine @ $1.841 \mathrm{ha}^{-1}$ singly or both in combination or any one of them in combination with one hand weeding reduced the dry weight of Joina by 72 to $92 \%$, Chechra by 66 to $76 \%$, Arail by 41 to $69 \%$ and Chanchi by 45 to $90 \%$. 
Table 4.Effected of weeding regime on dry weight of individual weed species.

\begin{tabular}{|c|c|c|c|c|c|c|c|c|c|c|}
\hline \multirow{2}{*}{$\begin{array}{l}\text { Weeding } \\
\text { regime }\end{array}$} & \multicolumn{10}{|c|}{ Weed density ( g.m -2) } \\
\hline & Joina & Matichaise & Nakphuli & Panichaise & Nolchaise & Cheehra & Angta & Khudeyshama & Anguleeghash & Barashama \\
\hline $\mathrm{W}_{0}$ & 3.06 & 5.89 & 3.38 & 1.01 & 0.62 & 1.61 & 35.29 & 1.18 & 4.62 & 0.71 \\
\hline $\mathrm{W}_{1}$ & $\begin{array}{l}1.56 \\
(-48.04)\end{array}$ & $\begin{array}{l}3.33 \\
(-43.46)\end{array}$ & $\begin{array}{l}2.85 \\
(-15.68)\end{array}$ & $\begin{array}{l}0.26 \\
(-74.26)\end{array}$ & - & $\begin{array}{l}0.37 \\
(-77.02)\end{array}$ & $\begin{array}{l}26.19 \\
(-25.79)\end{array}$ & $\begin{array}{l}0.56 \\
(-52.54)\end{array}$ & $\begin{array}{l}2.41 \\
(-47.84)\end{array}$ & - \\
\hline $\mathrm{W}_{2}$ & $\begin{array}{l}1.30 \\
(-57.52)\end{array}$ & $\begin{array}{l}2.13 \\
(-63.84)\end{array}$ & $\begin{array}{l}1.35 \\
(-60.00)\end{array}$ & $\begin{array}{l}0.20 \\
(-80.20)\end{array}$ & - & $\begin{array}{l}0.78 \\
(-51.55)\end{array}$ & $\begin{array}{l}21.09 \\
(-40.24)\end{array}$ & $\begin{array}{l}0.45 \\
(-38.14)\end{array}$ & $\begin{array}{l}2.02 \\
(-56.28)\end{array}$ & - \\
\hline $\mathrm{W}_{3}$ & $\begin{array}{l}0.72 \\
(-76.47)\end{array}$ & $\begin{array}{l}2.00 \\
(-66.04)\end{array}$ & $\begin{array}{l}1.20 \\
(-64.50)\end{array}$ & $\begin{array}{l}0.17 \\
(-83.17)\end{array}$ & - & $\begin{array}{l}0.26 \\
(-83.85)\end{array}$ & $\begin{array}{l}14.76 \\
(-58.18)\end{array}$ & $\begin{array}{l}0.38 \\
(-67.80)\end{array}$ & $\begin{array}{l}0.90 \\
(-80.52)\end{array}$ & - \\
\hline $\mathrm{W}_{4}$ & $\begin{array}{l}0.26 \\
(-91.50)\end{array}$ & $\begin{array}{l}0.72 \\
(-87.78)\end{array}$ & $\begin{array}{l}1.45 \\
(-57.10)\end{array}$ & - & $\begin{array}{l}0.24 \\
(-61.29)\end{array}$ & $\begin{array}{l}0.54 \\
(-66.46)\end{array}$ & $\begin{array}{l}10.66 \\
(-69.79)\end{array}$ & $\begin{array}{l}0.18 \\
(-84.75)\end{array}$ & $\begin{array}{l}0.45 \\
(-90.26)\end{array}$ & - \\
\hline $\mathrm{W}_{5}$ & $\begin{array}{l}0.80 \\
(-83.60)\end{array}$ & $\begin{array}{l}1.19 \\
(-79.82)\end{array}$ & $\begin{array}{l}1.24 \\
(-63.31)\end{array}$ & $\begin{array}{l}0.60 \\
(-40.59)\end{array}$ & $\begin{array}{l}0.49 \\
(-20.97)\end{array}$ & $\begin{array}{l}0.49 \\
(-69.57)\end{array}$ & $\begin{array}{l}12.97 \\
(-63.35)\end{array}$ & $\begin{array}{l}1.27 \\
(+7.63)\end{array}$ & $\begin{array}{l}0.43 \\
(-90.69)\end{array}$ & - \\
\hline $\mathrm{W}_{6}$ & $\begin{array}{l}0.23 \\
(-92.48)\end{array}$ & $\begin{array}{l}0.67 \\
(-88.62)\end{array}$ & $\begin{array}{l}1.72 \\
(-49.11)\end{array}$ & - & $\begin{array}{l}0.38 \\
(-38.71)\end{array}$ & $\begin{array}{l}0.51 \\
(-68.32)\end{array}$ & $\begin{array}{l}10.26 \\
(-70-93)\end{array}$ & $\begin{array}{l}0.17 \\
(-85.59)\end{array}$ & $\begin{array}{l}0.44 \\
(-90.48)\end{array}$ & - \\
\hline $\mathrm{W}_{7}$ & $\begin{array}{l}0.24 \\
(-92.16)\end{array}$ & $\begin{array}{l}0.43 \\
(-92.70)\end{array}$ & $\begin{array}{l}0.68 \\
(-79.88)\end{array}$ & - & - & $\begin{array}{l}0.47 \\
(-70.81)\end{array}$ & $\begin{array}{l}8.21 \\
(-76.74)\end{array}$ & $\begin{array}{l}0.17 \\
(-85.99)\end{array}$ & $\begin{array}{l}0.74 \\
(-83.98)\end{array}$ & - \\
\hline $\mathrm{W}_{8}$ & $\begin{array}{l}0.85 \\
(-72.22)\end{array}$ & $\begin{array}{l}0.83 \\
(-85.91)\end{array}$ & $\begin{array}{l}1.74 \\
(-48.52)\end{array}$ & $\begin{array}{l}0.46 \\
(-54.46)\end{array}$ & - & $\begin{array}{l}0.38 \\
(-76.40)\end{array}$ & $\begin{array}{l}12.14 \\
(-65.60)\end{array}$ & $\begin{array}{l}0.79 \\
(-33.05)\end{array}$ & $\begin{array}{l}0.41 \\
(-91.13)\end{array}$ & - \\
\hline $\mathrm{W}_{9}$ & - & - & - & - & - & - & - & - & - & - \\
\hline
\end{tabular}

Table 4. Continued

\begin{tabular}{|c|c|c|c|c|c|c|c|c|c|c|}
\hline \multirow[b]{2}{*}{$\begin{array}{l}\text { Weeding } \\
\text { regime }\end{array}$} & \multicolumn{10}{|c|}{ Weed density ( g.m -2) } \\
\hline & Arail & Chelaghash & Durba & Chanchi & Malancha & Keshuti & Helencha & Kanainala & Kanaibashi & Chhotodhudia \\
\hline $\mathrm{W}_{0}$ & 2.31 & 0.59 & 1.10 & 2.10 & 0.72 & 0.40 & 0.65 & 7.24 & $\begin{array}{l}0.78 \\
\end{array}$ & 0.86 \\
\hline $\mathrm{W}_{1}$ & $\begin{array}{l}1.19 \\
(-48.48)\end{array}$ & $\begin{array}{l}0.81 \\
(+37.29)\end{array}$ & - & $\begin{array}{l}1.29 \\
(-38.59)\end{array}$ & $\begin{array}{l}0.33 \\
(-54.17)\end{array}$ & $\begin{array}{l}0.21 \\
(-47.50)\end{array}$ & $\begin{array}{l}0.35 \\
(-46.15)\end{array}$ & $\begin{array}{l}5.04 \\
(-30.39)\end{array}$ & - & - \\
\hline $\mathrm{W}_{2}$ & $\begin{array}{l}1.01 \\
(-56.28)\end{array}$ & $\begin{array}{l}0.73 \\
(+23.73)\end{array}$ & - & $\begin{array}{l}1.20 \\
(-42.86)\end{array}$ & $\begin{array}{l}0.48 \\
(-33.33)\end{array}$ & $\begin{array}{l}0.38 \\
(-5.00)\end{array}$ & $\begin{array}{l}0.26 \\
(-60.00)\end{array}$ & $\begin{array}{l}3.32 \\
(-54.14)\end{array}$ & - & - \\
\hline $\mathrm{W}_{3}$ & $\begin{array}{l}0.44 \\
(-80.95)\end{array}$ & $\begin{array}{l}0.21 \\
(-64.41)\end{array}$ & - & $\begin{array}{l}0.83 \\
(-60.48)\end{array}$ & - & - & $\begin{array}{l}0.23 \\
(-64.62 \\
\end{array}$ & $\begin{array}{l}2.74 \\
(-62.15)\end{array}$ & - & - \\
\hline $\mathrm{W}_{4}$ & $\begin{array}{l}0.93 \\
(-59.74)\end{array}$ & $\begin{array}{l}0.35 \\
(-40.68)\end{array}$ & $\begin{array}{l}0.21 \\
(-80.91)\end{array}$ & $\begin{array}{l}0.81 \\
(-61.42)\end{array}$ & $\begin{array}{l}0.17 \\
(-76.39)\end{array}$ & - & - & $\begin{array}{l}1.17 \\
(-83.84)\end{array}$ & - & - \\
\hline $\mathrm{W}_{5}$ & 0.85 & 0.49 & 0.04 & 0.24 & - & 0.18 & - & 0.16 & - & - \\
\hline
\end{tabular}




\begin{tabular}{|c|c|c|c|c|c|c|c|c|c|c|}
\hline \multirow{2}{*}{$\begin{array}{l}\text { Weeding } \\
\text { regime }\end{array}$} & \multicolumn{10}{|c|}{ Weed density ( g.m -2) } \\
\hline & Arail & Chelaghash & Durba & Chanchi & Malancha & Keshuti & Helencha & Kanainala & Kanaibashi & Chhotodhudia \\
\hline & $(-63.20)$ & $(-16.95)$ & $(-96.36)$ & $(-88.57)$ & & $(-55.00)$ & & $(-97.79)$ & & \\
\hline $\mathrm{W}_{6}$ & $\begin{array}{l}1.35 \\
(-41.56)\end{array}$ & $\begin{array}{l}0.38 \\
(-35.59)\end{array}$ & - & $\begin{array}{l}0.21 \\
(-90.00)\end{array}$ & - & - & - & $\begin{array}{l}0.54 \\
(-92.54)\end{array}$ & - & - \\
\hline $\mathrm{W}_{7}$ & $\begin{array}{l}0.71 \\
(-69.26)\end{array}$ & $\begin{array}{l}0.57 \\
(-3.39)\end{array}$ & - & $\begin{array}{l}1.15 \\
(-45.24)\end{array}$ & - & - & - & $\begin{array}{l}1.14 \\
(-84.25)\end{array}$ & - & - \\
\hline $\mathrm{W}_{8}$ & $\begin{array}{l}1.35 \\
(-41.56)\end{array}$ & $\begin{array}{l}0.41 \\
(-30.51)\end{array}$ & - & $\begin{array}{l}0.21 \\
(-90.00)\end{array}$ & - & $\begin{array}{l}0.11 \\
(-72.50)\end{array}$ & - & $\begin{array}{l}0.83 \\
(-88.24)\end{array}$ & - & - \\
\hline$\overline{\mathrm{W}_{9}}$ & - & - & - & - & - & - & - & -- & - & - \\
\hline
\end{tabular}

\section{Table 4. Continued}

\begin{tabular}{|c|c|c|c|c|c|c|c|c|}
\hline \multirow{2}{*}{$\begin{array}{l}\text { Weeding } \\
\text { regime }\end{array}$} & \multicolumn{8}{|c|}{ Weed dry weight $\left(\mathrm{gm}^{-2}\right)$} \\
\hline & Baradhudia & Panikachu & Panilong & Amrulshak & Shusnishak & Panimarich & Acidghash & Kalmilata \\
\hline $\mathrm{W}_{0}$ & 0.42 & 1.81 & 1.41 & 0.92 & 1.68 & 0.79 & 0.82 & 1.06 \\
\hline$\overline{W_{1}}$ & - & $1.38(-23.76)$ & $0.79(-43.97)$ & $1.01(+9.78)$ & $1.14(-32.14)$ & $0.72(-8.86)$ & $0.42(-48.78)$ & $0.78(-26.42)$ \\
\hline $\mathrm{W}_{2}$ & - & $0.42(-76.80)$ & $0.20(-85.82)$ & $0.39(-57.61)$ & $0.82(-51.19)$ & $0.55(-30.98)$ & - & - \\
\hline$\overline{W_{3}}$ & - & $0.35(-80.00)$ & $0.18(-87.23)$ & $0.32(-65.22)$ & $0.37(-77.98)$ & $0.07(-91.14)$ & - & - \\
\hline $\mathrm{W}_{4}$ & - & - & - & $0.24(-73.91)$ & $0.02(-98.81$ & $0.04(-94.94)$ & - & - \\
\hline $\mathrm{W}_{5}$ & - & - & $0.62(-56.03)$ & - & $0.13(-92.26)$ & $0.11(-86.08)$ & - & $0.04(-96.23)$ \\
\hline $\mathrm{W}_{6}$ & - & - & - & - & - & - & - & - \\
\hline $\mathrm{W}_{7}$ & - & - & - & - & - & $0.04(-94.94)$ & - & - \\
\hline $\mathrm{W}_{8}$ & - & - & $0.15(-89.36)$ & - & $0.20(-88.10)$ & $0.03(-96.20)$ & - & - \\
\hline $\mathrm{W}_{9}$ & - & - & - & - & - & - & - & - \\
\hline
\end{tabular}

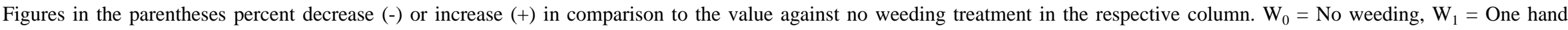

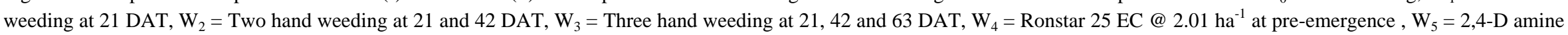

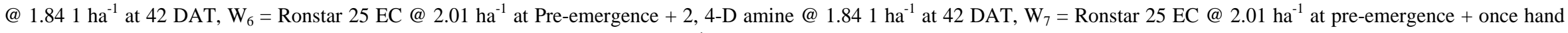
weeding at $42 \mathrm{DAT}, \mathrm{W}_{8}=$ One hand weeding at $21 \mathrm{DAT}+2$, 4-amine @ $1.841 \mathrm{ha}^{-1}$ at $42 \mathrm{DAT}$ and $\mathrm{W}_{9}=$ Weed free. 
The Phytotoxicity of pre-emergence application of Ronstar 25 EC@ 2.01 ha $^{-1}$ marked a rating of 2.0 while that of post -emergence application of 2,4-D amine @ 1.841 ha $^{-1}$ marked a rating of 1.3. Thus, Ronstar 25 EC @ 2.01 ha $^{-1}$ was slightly more toxic than 2, 4-D amine @ 1.841 ha $^{-1}$. In Ronstar 25 EC@ 2.01 ha $^{-1}$ applied plots rice plants showed slight yellowing of leaves, burning of leaf-tips and temporary stunting of the growth and the plants required 8 to 10 days to recover and resume normal growth and color. On the other hand, in 2, 4-D amine @ 1.841 ha $^{-1}$ treated plots the rice plants showed only slight yellowing of some leaves which resumed normal color within 5 to 7 days. A slightly higher toxicity of rice plants to Ronstar 25 EC @ 2.01 ha $^{-1}$ than 2, 4-D amine @ $1.841 \mathrm{ha}^{-1}$ might be due to the fact that Ronstar 25 EC was applied pre-emergence and it acted on the emerging, young rice seedlings while 2, 4-D amine @ $1.841 \mathrm{ha}^{-1}$ was applied post emergence at 42 days after transplanting of the rice seedling. Plant height, total number of tillers hill $^{-1}$, number of ear bearing tillers hill-1 ${ }^{-1}$ number of non ear bearing tillers hill ${ }^{-1}$, number of grains panicle $^{-1}$, number of sterile spike lets grains panicle ${ }^{-1}$, grain yield, straw yield, biological yield and harvest index were significantly affected by weeding regime. Plant height in crops hand weeded once, twice or thrice, or chemically weeded by Ronstar 25 EC @ 2.01 ha $^{-1}$ or 2, 4-D amine @ 1.84 $1 \mathrm{ha}^{-1}$ or by both, or weeded by a combination of hand weeding and chemical weeding was statistically similar but significantly higher than that in crops receiving no weeding (Table 5). In short, it may be said that the unwedded crops were shorter than the weeded crops. The crop kept weed free throughout the life cycle produced the highest total number of tillers hill ${ }^{-1}$ and the crop kept unwedded throughout the life cycle produced the lowest number of tillers hill-1 ${ }^{-1}$ Table 5), the difference in the total number of tillers hill ${ }^{-1}$ between these two treatments being significant. However, the total number of tiller hill $^{-1}$ in crops hand weeded twice, hand weeded thrice, weeded by Ronstar 25 EC @ 2.01 ha $^{-1}$, weeded by Ronstar 25 EC @ 2.01 ha $^{-1}$ in combination with one hand weeding and the crops kept weed free were statistically identical. Similarly, the crops kept unwedded, hand weeded by hand weeding once, Ronstar 25 EC @ 2.01 ha $^{-1}+2$, 4-D amine @ $1.841 \mathrm{ha}^{-1}$, Ronstar 25 EC @ $2.01 \mathrm{ha}^{-1}+2$, 4-D amine @ $1.841 \mathrm{ha}^{-1}$, and one hand weeding + 2, 4D amine @ 1.841 ha $^{-1}$ produced statistically similar total number of tillers hill ${ }^{-1}$. The number of ear bearing tillers hill ${ }^{-1}$ in weed free crop being identical with that in crop weeded by Ronstar 25 EC @ $2.01 \mathrm{ha}^{-1}+$ one hand weeding was significantly higher than those in rest of the weeding treatments (Table 5). Three hand weeding, Ronstar 25 EC @ 2.01 ha $^{-1}$, 2, 4-D amine @ 1.841 ha 
1, Ronstar 25 EC @ 2.01 ha $^{-1}+$ 2, 4-D amine @ 1.841 ha $^{-1}$, and Ronstar 25 EC @ 2.01 ha $^{-1}+2$, 4D amine @ $1.841 \mathrm{ha}^{-1}$, and Ronstar 25 EC @ $2.01 \mathrm{ha}^{-1}+$ one hand weeding produced statistically similar number of ear bearing tiller hill ${ }^{-1}$, Similarly, the crop hand weeded twice, hand weeded thrice, and weeded by Ronstar 25 EC @ 2.01 ha $^{-1}$,2, 4-D amine @ 1.841 ha $^{-1,}$, Ronstar 25 EC @ 2.01 ha $^{-1}+2$, 4-D amine @ 1.841 ha $^{-1}$ and one hand weeding + 2, 4-D amine @ 1.841 ha $^{-1}$ produced statistically identical number of car bearing tillers hill ${ }^{-1}$. The number of ear bearing tiller hill $^{-1}$ in one hand weeding, two hand weeding, three hand weeding, and weeded by 2, 4-D amine @ $1.841 \mathrm{ha}^{-1}$ singly or in combination with one hand weeding being at par was significantly higher than that in unwedded crop and lower than that in weed free crop. However, the number of ear bearing tillers hill ${ }^{-1}$ was highest in crop kept weed free and lowest in crop kept unwedded. No weeding, one hand weeding, two hand weeding, three hand weeding, three hand weeding and Ronstar 25 EC @ 2.01 ha $^{-1}+$ one hand weeding treatments produced statistically identical number of non ear bearing tillers hill $^{-1}$ (Table 5). It was also statistically similar in one hand weeding, two hand weeding, three hand weeding, Ronstar 25 EC @ 2.01 ha-1, 2,4-D amine @ 1.841 ha $^{-1}$, Ronstar 25 EC @ 2.01 ha $^{-1}+$ one hand weeding, one hand weeding + 2, 4-D amine @ 1.841 ha $^{-1}$ and weed free treatments. However, the number of non -ear bearing tillers hill ${ }^{-1}$ was highest in no weeding treatment and the lowest in the Ronstar 25 EC @ 2.01 ha $^{-1}+2,4-\mathrm{D}$ amine @ 1.841 ha $^{-1}$ treatment, the difference between these two treatments being significant. Weeding regime had no significant effect on the panicle length but it was numerically largest in weed free treatment and smallest in no weeding treatment (Table5). Number of grains panicle ${ }^{-1}$ in no weeding, one hand weeding, two hand weeding, three hand weeding, Ronstar 25 EC @ 2.01 ha $^{-1}$, 2, 4-D amine @ $1.841 \mathrm{ha}^{-1}$ treatments was statistically similar (Table 5). Except that in no weeding treatment, which produced the lowest, and in weed free treatment which produced the highest, the number of grains panicle ${ }^{-1}$ was statistically identical in all other treatments. 
Table 5 Effect of weeding regime on the crop characters of transplant aman rice (BRII)

\begin{tabular}{|c|c|c|c|c|c|c|c|c|c|c|c|c|}
\hline Weeding regime & $\begin{array}{l}\text { Plant } \\
\text { height } \\
(\mathrm{cm})\end{array}$ & $\begin{array}{l}\text { Total tillers } \\
\text { hill }^{-1} \text { (no) }\end{array}$ & $\begin{array}{l}\text { Ear bearing } \\
\text { tillers hill }{ }^{-1} \\
\text { (no) }\end{array}$ & $\begin{array}{l}\text { Non-ear } \\
\text { bearing } \\
\text { tillers hill- } \\
{ }^{1}(\mathrm{no})\end{array}$ & $\begin{array}{l}\text { Panicle } \\
\text { length } \\
\text { (cm) }\end{array}$ & $\begin{array}{l}\text { Grains } \\
\text { panicle }^{-1} \\
\text { (no) }\end{array}$ & $\begin{array}{l}\text { Sterile } \\
\text { spikelets } \\
\text { panicle (no) }\end{array}$ & $\begin{array}{l}1000 \text { grain } \\
\text { weight (g) }\end{array}$ & $\begin{array}{l}\text { Grain yield } \\
\left(\mathrm{t} \mathrm{ha}^{-1}\right)\end{array}$ & $\begin{array}{l}\text { Straw yield } \\
\text { (t ha-1) }\end{array}$ & $\begin{array}{l}\text { Biological } \\
\text { yield (t ha- } \\
{ }^{1} \text { ) }\end{array}$ & $\begin{array}{l}\text { Harvest } \\
\text { index (\%) }\end{array}$ \\
\hline No weeding $\left(\mathrm{W}_{0}\right)$ & $\begin{array}{l}105.3 b \\
(-11.96)\end{array}$ & $\begin{array}{l}11.57 \mathrm{~d} \\
(-11.75)\end{array}$ & $\begin{array}{l}7.65 e \\
(-26.58)\end{array}$ & $\begin{array}{l}3.92 \mathrm{a} \\
(+45.72)\end{array}$ & $\begin{array}{l}22.20 \\
(-6.61)\end{array}$ & $\begin{array}{l}79.55 c \\
(-15.63)\end{array}$ & $\begin{array}{l}28.43 a \\
(+33.16)\end{array}$ & $\begin{array}{l}22.15 \\
(-1.34)\end{array}$ & $\begin{array}{l}2.17 \mathrm{c} \\
(-54.51)^{*}\end{array}$ & $\begin{array}{l}4.27 d \\
(-40.45)\end{array}$ & $\begin{array}{l}6.44 \mathrm{c} \\
(-46.06)\end{array}$ & $\begin{array}{l}33.66 \mathrm{c} \\
(-15.58)\end{array}$ \\
\hline $\begin{array}{l}\text { One hand weeding at } 21 \\
\text { DAT }\left(\mathrm{W}_{1}\right)\end{array}$ & $\begin{array}{l}116.8 \mathrm{a} \\
(-2.34)\end{array}$ & $\begin{array}{l}11.98 \mathrm{~cd} \\
(-8.62)\end{array}$ & $\begin{array}{l}8.54 d \\
(-18.04)\end{array}$ & $\begin{array}{l}3.45 \mathrm{abc} \\
(+28.25)\end{array}$ & $\begin{array}{l}22.85 \\
(-3.26)\end{array}$ & $83.07 \mathrm{bc}$ & $\begin{array}{l}26.64 \mathrm{ab} \\
(+24.78)\end{array}$ & $\begin{array}{l}22.16 \\
(-1.29)\end{array}$ & $\begin{array}{l}4.05 b \\
(-15.09)\end{array}$ & $\begin{array}{l}6.33 c \\
(-11.72)\end{array}$ & $\begin{array}{l}10.38 \mathrm{~b} \\
(-12.98)\end{array}$ & $\begin{array}{l}38.90 \mathrm{ab} \\
(-2.43)\end{array}$ \\
\hline $\begin{array}{l}\text { Two hand weeding at } 21 \\
\text { and } 42 \mathrm{DAT}\left(\mathrm{W}_{2}\right)\end{array}$ & $\begin{array}{l}118.0 \mathrm{a} \\
(-1.34)\end{array}$ & $\begin{array}{l}12.44 \mathrm{abc} \\
(-5.11)\end{array}$ & $\begin{array}{l}8.96 \mathrm{~cd} \\
(-14.01)\end{array}$ & $\begin{array}{l}\begin{array}{l}3.48 \mathrm{ab} \\
(+29.37)\end{array} \\
\end{array}$ & $\begin{array}{l}22.93 \\
(-2.92)\end{array}$ & $\begin{array}{l}\text { 85.71abc } \\
(-9.10)\end{array}$ & $\begin{array}{l}24.51 \mathrm{bc} \\
(+14.80)\end{array}$ & $\begin{array}{l}22.48 \\
(+0.13)\end{array}$ & $\begin{array}{l}4.09 b \\
(-14.26)\end{array}$ & $\begin{array}{l}6.50 \mathrm{bc} \\
(-9.34)\end{array}$ & $\begin{array}{l}10.59 b \\
(-11.31)\end{array}$ & $\begin{array}{l}38.54 a b \\
(-3.34)\end{array}$ \\
\hline $\begin{array}{l}\text { There hand weeding at } \\
21,42 \text { and } 63 \text { DAT }\left(W_{3}\right)\end{array}$ & $\begin{array}{l}118.5 a \\
(-0.92)\end{array}$ & $\begin{array}{l}\text { 12.53abc } \\
(-4.42)\end{array}$ & $\begin{array}{l}\text { 9.19bcd } \\
(-11.80)\end{array}$ & $\begin{array}{l}3.37 \mathrm{abc} \\
(+25.28)\end{array}$ & $\begin{array}{l}22.63 \\
(-4.19)\end{array}$ & $\begin{array}{l}86.95 \mathrm{abc} \\
(-7.78)\end{array}$ & $\begin{array}{l}\begin{array}{l}\text { 23.71bcd } \\
(+11.05)\end{array} \\
\end{array}$ & $\begin{array}{l}22.53 \\
(+0.36)\end{array}$ & $\begin{array}{l}4.13 \mathrm{~b} \\
(-13.42)\end{array}$ & $\begin{array}{l}6.61 \mathrm{bc} \\
(-7.81)\end{array}$ & $\begin{array}{l}10.73 b \\
(-10.13)\end{array}$ & $\begin{array}{l}38.36 \mathrm{ab} \\
(-3.79)\end{array}$ \\
\hline $\begin{array}{l}\text { Ronstar } 25 \text { EC @ } 2.01 \text { ha } \\
{ }^{1} \text { at pre-emergence }\left(\mathrm{W}_{4}\right)\end{array}$ & $\begin{array}{l}117.8 \mathrm{a} \\
(-1.51)\end{array}$ & $\begin{array}{l}\text { 12.31abcd } \\
(-6.10)\end{array}$ & $\begin{array}{l}9.46 \mathrm{bc} \\
(-9.21)\end{array}$ & $\begin{array}{l}2.85 \mathrm{bc} \\
(+5.95)\end{array}$ & $\begin{array}{l}22.74 \\
(-3.73)\end{array}$ & $\begin{array}{l}86.94 \mathrm{abc} \\
(-7.80)\end{array}$ & $\begin{array}{l}24.54 \mathrm{bc} \\
(+14.94)\end{array}$ & $\begin{array}{l}22.30 \\
(-0.67)\end{array}$ & $\begin{array}{l}4.21 \mathrm{~b} \\
(-11.74)\end{array}$ & $\begin{array}{l}6.65 \mathrm{bc} \\
(-7.25)\end{array}$ & $\begin{array}{l}10.86 \mathrm{~b} \\
(-9.05)\end{array}$ & $\begin{array}{l}38.67 \mathrm{ab} \\
(-3.01)\end{array}$ \\
\hline $\begin{array}{l}\text { 2, 4-D amine @ } 1.841 \text { ha } \\
{ }^{1} \text { at } 42 \mathrm{DAT}\left(\mathrm{W}_{5}\right)\end{array}$ & $\begin{array}{l}117.2 \mathrm{a} \\
(-2.01)\end{array}$ & $\begin{array}{l}12.11 \mathrm{bcd} \\
(-7.63)\end{array}$ & $\begin{array}{l}9.26 b c d \\
(-11.13)\end{array}$ & $\begin{array}{l}2.83 \mathrm{bc} \\
(+5.20)\end{array}$ & $\begin{array}{l}22.25 \\
(-5.80)\end{array}$ & $\begin{array}{l}\text { 85.95abc } \\
(-8.85)\end{array}$ & $\begin{array}{l}\text { 26.51ab } \\
(+24.17)\end{array}$ & $\begin{array}{l}22.53 \\
(+0.36)\end{array}$ & $\begin{array}{l}3.98 \mathrm{~b} \\
(-15.51)\end{array}$ & $\begin{array}{l}6.67 b c \\
(-6.97)\end{array}$ & $10.64 \mathrm{~b}$ & $\begin{array}{l}37.16 \mathrm{~b} \\
(-6.80)\end{array}$ \\
\hline $\begin{array}{l}\text { Ronstar } 25 \text { EC @ } 2.01 \text { ha } \\
1 \text { at pre-emergence }+2 \text {, 4- } \\
\text { D amine @ } 1.841 \text { ha }^{-1} \text { at } \\
42 \text { DAT }\left(\mathrm{W}_{6}\right)\end{array}$ & $\begin{array}{l}117.7 \mathrm{a} \\
(-1.59)\end{array}$ & $\begin{array}{l}11.99 \mathrm{~cd} \\
(-8.54)\end{array}$ & $\begin{array}{l}9.43 b c \\
(-9.50)\end{array}$ & $\begin{array}{l}2.56 c \\
(-4.83)\end{array}$ & $\begin{array}{l}22.78 \\
(-3.56)\end{array}$ & $\begin{array}{l}\text { 87.71abc } \\
(-6.98)\end{array}$ & $\begin{array}{l}\begin{array}{l}24.61 b c \\
(+15.27)\end{array}\end{array}$ & $\begin{array}{l}22.40 \\
(-0.22)\end{array}$ & $\begin{array}{l}4.24 \mathrm{~b} \\
(-11.11)\end{array}$ & $\begin{array}{l}\text { 6.43c } \\
(-10.32)\end{array}$ & $\begin{array}{l}10.67 b \\
(-10.64)\end{array}$ & $\begin{array}{l}39.68 \mathrm{a} \\
(-0.48)\end{array}$ \\
\hline $\begin{array}{l}\text { Ronstar } 25 \text { EC @ } 2.01 \text { ha } \\
{ }^{1} \text { at pre-emergence + one } \\
\text { hand weeding at } 42 \text { DAT } \\
\left(\mathrm{W}_{7}\right)\end{array}$ & $\begin{array}{l}118.5 a \\
(-0.92)\end{array}$ & $\begin{array}{l}12.89 \mathrm{ab} \\
(-1.68)\end{array}$ & $\begin{array}{l}9.82 \mathrm{ab} \\
(-5.76)\end{array}$ & $\begin{array}{l}3.07 a b c \\
(+14.13)\end{array}$ & $\begin{array}{l}23.43 \\
(-0.80)\end{array}$ & $\begin{array}{l}92.08 \mathrm{ab} \\
(-2.38)\end{array}$ & $\begin{array}{l}22.09 \mathrm{~cd} \\
(+3.47)\end{array}$ & $\begin{array}{l}22.44 \\
(-0.04)\end{array}$ & $\begin{array}{l}4.67 a \\
(-2.10)\end{array}$ & $\begin{array}{l}6.87 a b \\
(-4.18)\end{array}$ & $\begin{array}{l}11.54 \mathrm{a} \\
(-3.35)\end{array}$ & $\begin{array}{l}40.38 \mathrm{a} \\
(+1.28)\end{array}$ \\
\hline $\begin{array}{l}\text { One hand weeding at } 21 \\
\text { DAT }+2,4-\mathrm{D} \text { amine @ } \\
1.8411 \text { ha }^{-1} \text { at } 42 \text { DAT } \\
\left(\mathrm{W}_{8}\right)\end{array}$ & $\begin{array}{l}117.4 \mathrm{a} \\
(-1.84)\end{array}$ & $\begin{array}{l}11.82 \mathrm{~cd} \\
(-9.84)\end{array}$ & $\begin{array}{l}8.87 \mathrm{~cd} \\
(-14.88)\end{array}$ & $\begin{array}{l}2.96 \mathrm{bc} \\
(+10.04)\end{array}$ & $\begin{array}{l}23.18 \\
(-1.86)\end{array}$ & $\begin{array}{l}90.34 \mathrm{ab} \\
(-4.19)\end{array}$ & $\begin{array}{l}23.06 \mathrm{~cd} \\
(+8.01)\end{array}$ & $\begin{array}{l}22.74 \\
(+1.29)\end{array}$ & $\begin{array}{l}4.15 b \\
(-13.00)\end{array}$ & $\begin{array}{l}6.34 \mathrm{c} \\
(-11.58)\end{array}$ & $\begin{array}{l}10.49 b \\
(-12.14)\end{array}$ & $\begin{array}{l}39.59 a \\
(-0.70)\end{array}$ \\
\hline Weed free $\left(\mathrm{W}_{9}\right)$ & $119.6 \mathrm{a}$ & $13.11 \mathrm{a}$ & $10.42 \mathrm{a}$ & $2.69 \mathrm{bc}$ & 23.62 & $94.29 \mathrm{a}$ & $21.35 \mathrm{~d}$ & 22.45 & $4.77 \mathrm{a}$ & $7.17 \mathrm{a}$ & $11.94 \mathrm{a}$ & $39.87 \mathrm{a}$ \\
\hline $\mathrm{S}_{\mathrm{X}}$ & 0.884 & 0.199 & 0.205 & 0.210 & - & 2.282 & 0.721 & - & 0.065 & 0.102 & 0.128 & 0.486 \\
\hline CV (\%) & 3.12 & 5.63 & 7.76 & 6.19 & 6.10 & 9.06 & 10.18 & 3.14 & 5.55 & 5.52 & 4.24 & 4.38 \\
\hline Level of Significance & 0.01 & 0.01 & 0.01 & 0.01 & NS & 0.01 & 0.01 & NS & 0.01 & 0.01 & 0.01 & 0.01 \\
\hline
\end{tabular}

In a column, figures having a common letter (s) do not differ significantly, NS = Not Significant, Figures in the parenthess indicate percent increase (+) or per cent decrease (-) in comparison to value against weed free treatments in the respective column. 
The crop not weeded at all produced the highest number of sterile spikelets panicle ${ }^{-1}$ and the crops kept weed free although produced the lowest number of sterile spikelets panicle ${ }^{-1}$, the difference between these two treatments ( in respect of production of number of sterile spikelets panicle ${ }^{-1}$ ) being significant (Table5). However, the number of sterile spikelets panicle ${ }^{-1}$ in weed free treatment was statistically at par with that in one hand weeding + 2, 4-D amine @ $1.841 \mathrm{ha}^{-1}$, Ronstar 25 EC @ 2.01 ha $^{-1}+$ one hand weeding and three hand weeding, and that in no weeding treatment was statistically similar with the number of sterile spikelets in one hand weeding and 2, 4-D amine @ 1.841 ha $^{-1}$ treatments. The number of sterile spikelets in rest of the treatments, that is, two hand weeding, three hand weeding, Ronstar 25 EC @ 2.01 ha $^{-1}$ and Ronstar 25 EC @ 2.01 $\mathrm{ha}^{-1}+$ 2, 4-D amine@1.84 $1 \mathrm{ha}^{-1}$ treatments was also indentical. 100- grain weight was not significantly affected by weeding regime or due to their interaction (Table 5) Grain yield was highest in weed free treatment but it was at par with that in Ronstar 25 EC@ 2.01 ha $^{-1}+$ one hand weeding treatment (Table 5). The lowest grain yield was produced by the crop not weeded at all. Grain yield in one hand weeding, two hand weeding, three hand weeding, Ronstar 25 EC @ 2.01 ha ${ }^{-1}$, 2, 4-D amine @ 1.841 ha $^{-1}$, Ronstar 25 EC @ 2.01 ha $^{-1}+2$, 4-D amine @ 1.841 ha $^{-1}$ and one hand weeding + 2, 4-D amine @ $1.841 \mathrm{ha}^{-1}$ treatments being statistically similar was significantly higher than that in no weeding and significantly lower than in weed or Ronstar 25 EC@ $2.01 \mathrm{ha}^{-1}+$ one hand weeding treatment. The highest straw yield was produced in weed free crop but it was identical with that produced in Ronstar 25 EC @ 2.01 ha $^{-1}+$ one hand weeding (Table 5). The lowest straw yield was produced in the crop receiving no weeding, the difference between the lowest and highest straw yields being significant. Straw yields in one hand weeding, two hand weeding, three hand weeding, Ronstar 25 EC @ 2.01 ha $^{-1}$, 2, 4-D amine @ 1.841 ha $^{-1}$, Ronstar 25 EC@2.01 ha-1 + 2, 4-D amine @ 1.841 ha $^{-1}+$ one hand weeding treatments were statistically identical. The highest biological yield was produced in weed free crop and it was at par with the biological yield in Ronstar 25 EC @ 2.01 ha $^{-1}+$ one hand weeding treatment and significantly higher than that in any other weeding treatments (Table 5). The lowest biological yield was produced in the crop not weeded at all and it was significantly lower than that in any other weeding treatments. The biological yield in one hand weeding was statistically similar with that in two hand weeding, three hand weeding, Ronstar 25 EC @ 2.01 ha $^{-1}$, 2, 4-D amine @ 1.84 1 ha $^{-1}$, Ronstar 25 EC @ 2.01 ha $^{-1}+$ 2, 4-D amine @ 1.841 ha $^{-1}$, Ronstar 25 EC @ 2.01 ha $^{-1}+2$, 4-D amine @1.84 1 ha $^{-1}$ and one hand weeding + 2, 4-D amine @ 1.841 ha $^{-1}$ treatments. Harvest index was highest in weed free crop but it was identical with that in one hand weeding, two hand handing, three hand weeding, three hand weeding, Ronstar 25 EC @ 2.01 ha $^{-1}$, Ronstar 25 EC @ $2.01 \mathrm{ha}^{-1}+$ 2, 4-D amine @ $1.841 \mathrm{ha}^{-1}$, Ronstar 25 EC @ 2.01 ha-1 + one hand weeding and one hand weeding + 2,4-D amine @ 1.841 ha $^{-1}$ (Table 5). In 2, 4-D amine @ 1.841 ha $^{-1}$, treated crop, harvest index was significantly higher than that in no weeding and significantly lower than that in Ronstar 25 EC @ 2.01 ha $^{-1}$, Ronstar 25 EC @ 2.01 ha $^{-1}+$ one hand weeding, one hand weeding + 2, 4-D amine @ 1.841 ha $^{-1}$ and weed free treatments. The lowest harvest index was produced in the crop not weeded at all. From the effects of weeding regime on the yield and yield contributing characters it is seen that, the crop which was not weeded at all producing smallest number of ear bearing tillers hill ${ }^{-1}$, smallest panicle, smallest number of grains hill ${ }^{-1}$ and the lightest grains produced significantly lowest grain yield while the crop kept weed free throughout the life cycle producing largest number of ear bearing tillers hill ${ }^{-1}$, largest panicle, largest number of grains panicle $^{-1}$ and the heaviest grains produced significantly highest grain yield. Similarly the crop kept unwedded although producing shortest plants and the smallest total number of tillers hill $^{-1}$ produced the lowest straw yield and the crop in which weeds were not allowed to grow at all (weed free) producing tallest plants and largest total number of tillers hill ${ }^{-1}$ produced significantly 
highest straw yield. This difference in the production of yield and yield contributing characters between the no weeding and weed free treatments was attributed to weed competition. In weed free treatment no weeds were allowed to grow, so the crop did not experience any competition from the weeds and this resulted to the production of superior-most yield and yield contributing characters. On the other hand, in no weeding treatment, the weeds were allowed to grow unrestricted up to the crop harvest and the crop experienced tremendous competition from these weeds through out the life cycle. And, probably this was the main reason for the consequence of reduction of grain and straw yields by $54.51 \%$ and $40.45 \%$, respectively, in the no weeding treatments. That the weeds reduce the grain yield of transplant aman rice by 7 to $80 \%$ has also been reported by other (BRRI, 1981; Sattar, 1986) and the extent yield reduction due to weed competition in transplant aman rice varies with factors like species, density, time of emergence and duration of competition of weeds, variety and density of the rice plants, soil fertility level and climate condition (Chang, 1970). Among the weed control treatments, Ronstar 25 EC @ 2.01 ha $^{-1}$ + one hand weeding controlled the weeds to the greatest extent, reducing weed density by $69.72 \%$ and weed dry weight by $82.47 \%$, and produced the yield and yield attributes statistically similar to those in weed free treatment. So it was considered to be best in terms of weed control and crop performance. Hand weeding once, twice or thrice produced statistically similar yield and yield attributes. So, no extra benefit in terms of grain and straw yields could be obtained in this study from weeding more than once. Ronstar 25 EC@ 2.01 ha $^{-1}$ and2, 4-D amine @ 1.841 ha $^{-1}$ singly or in combination and 2, 4-D amine @ $1.841 \mathrm{ha}^{-1}+$ one hand weeding also produced statistically identical grain and straw yields. So all of these weed control measures were also of similar effect in producing grain and straw yields. So, considering the weed control efficiency and agroeconomic performance of transplant aman rice in respect of weeding regime, in the context of the present study, recommendation should go for weed control by Ronstar 25 EC @ 2.01 ha $^{-1}+$ One hand weeding at 42 DAT. If, however, herbicide is not available and the weeds to be controlled by hand weeding, then one hand weeding at 21 DAT is recommended. On the other hand, where there is scarcity of labor and the situation demands the use of herbicide for controlling the weeds, one should go for controlling weeds by Ronstar 25 EC @ 2.01 ha $^{-1}$ at pre-emergence.

\section{REFERENCES}

BBS (Bangladesh Bureau of Statistics) 2008. Yearbook of Agricultural Statistics of Bangladesh. Bangladesh Bureau of Statistics, Stat. Div., Min. Plan., Govt. People's Repub. Bangladesh. p.45.

BRRI (Bangladesh Rice Research Institute)1981. Annwal Report for 1977-78. Bangladesh Rice Res.Inst., Joydebpur, Gazipur. pp. 11-19.

Chang, W.L. 1970. The effect of weeds on rice in paddy field I. Weed species and population density. J.Taiwan Agric. 19(4): 18-25.

Kim, S.C.; Heu, H. and Chung, K.Y, 1975. Ecological aspect of some perennial weeds and its effective control in paddy rice, Memorial Res. $10^{\text {th }}$ Anniv. Yungnam Crop Exp. Sta. 1: 34-44.

Mamun. A.A. 1988. Crop -ecosystem, weed vegetation and weed management in Dakshin chamuri and Jawar. In: Proc. Mid-term Review workshop of JSARD, 24 January, 1988. JICA, Gulshan, Dhaka. Bangladesh.pp. 316-342.

Sattar, S.A. 1986. Strategics for weed management in rice in Bangladesh. In: Adv.Agron. Res. Bangladesh. Bangladesh Soc. Agron. 1: 41-47. 